\title{
Development of the VOILA LIBS instrument for volatiles scouting in polar regions of the Moon
}

Lutz Richter, Michael Deiml, Markus Glier, Albert Althammer, Mattia Reganaz, et al.

Lutz Richter, Michael Deiml, Markus Glier, Albert Althammer, Mattia Reganaz, Stefan Spiekermann, David Mendoza Gandara, Heinz-Wilhelm Huebers, Peter Weßels, Jörg Neumann, David Sebastian Vogt, Susanne Schröder, "Development of the VOILA LIBS instrument for volatiles scouting in polar regions of the Moon," Proc. SPIE 11852, International Conference on Space Optics - ICSO 2020, 118521I (11 June 2021); doi: 10.1117/12.2599244

SPIE Event: International Conference on Space Optics - ICSO 2021, 2021, Online Only 


\section{International Conference on Space Optics-ICSO 2020}

Virtual Conference

30 March-2 April 2021

Edited by Bruno Cugny, Zoran Sodnik, and Nikos Karafolas
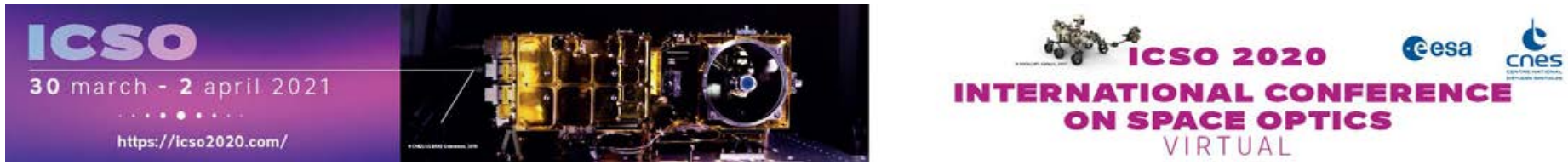

\section{Development of the VOILA LIBS instrument for volatiles scouting in polar regions of the Moon}

\section{Cesa isoporecestings denes}




\title{
Development of the VOILA LIBS instrument for volatiles scouting in polar regions of the Moon
}

\author{
Lutz Richter*a, Michael Deiml ${ }^{\mathrm{a}}$, Markus Glier ${ }^{\mathrm{a}}$, Albert Althammer ${ }^{\mathrm{a}}$, Mattia Reganaz ${ }^{\mathrm{a}}$, Stefan \\ Spiekermann $^{\mathrm{b}}$, David Mendoza Gandara ${ }^{\mathrm{b}}$, Heinz-Wilhelm Huebers ${ }^{\mathrm{c}}$, Peter Weßels ${ }^{\mathrm{b}}$, Jörg Neumann ${ }^{\mathrm{b}}$, \\ David Sebastian Vogt ${ }^{\mathrm{c}}$, Susanne Schröder ${ }^{\mathrm{c}}$ \\ ${ }^{a}$ OHB System AG, 1 Manfred-Fuchs-Strasse, Wessling, D-82234 Wessling, Germany; ${ }^{b}$ Laser \\ Zentrum Hannover e.V. (LZH), 8 Hollerithallee, D-30419 Hannover, Germany; ${ }^{\circ}$ Deutsches Zentrum \\ fuer Luft- und Raumfahrt e.V., Institut fuer Optische Sensorsysteme, 2 Rutherfordstrasse, D-12489 \\ Berlin, Germany
}

\begin{abstract}
In this paper, we report on the on-going development of a compact analytical instrument for future missions to the Moon, using the LIBS technique. The instrument, christened VOILA (Volatiles Identification by Laser Ablation), is part of the "LUVMI-X" scenario funded by the European Union's Horizon 2020 programme which envisions the concept of a rover carrying a suite of instruments for detecting and characterizing volatiles as a component of lunar soil ("regolith") at high latitudes on the Moon. Behaviour of the plasma created by LIBS target ablation depends on the prevalent atmospheric pressure. Mars atmospheric pressure is close to ideal for the LIBS technique. On bodies without atmosphere such as the Moon, the lack of confining pressure leads to the plasma dissipating quickly causing weaker signals. India has developed the first, modest LIBS instrument for operation on the Moon with however a number of limitations in its optical design. Moreover, the lander of the corresponding mission Chandrayaan-2 crashed during landing in 2019. Our VOILA is designed to be between the quite capable but large and massive Mars LIBS instruments of NASA (ChemCam on the CURIOSITY rover, and the follow-on SuperCam on the PERSEVERANCE rover), and the very modest Indian LIBS for Chandrayaan2. The VOILA working range will vary between 0.3 and $0.5 \mathrm{~m}$, with a laser pulse energy of $\sim 15 \mathrm{~mJ}$.
\end{abstract}

Keywords: LIBS, Moon, volatiles, plasma, laser, optical head

\section{INTRODUCTION}

The lunar South Pole is of great interest for upcoming lunar exploration endeavors due to the inference of large reservoirs of water ice in the pole's permanently shadowed regions, using different observational techniques from lunar orbit [1]. Water is an important resource for life support on future Moon bases and for potential applications as fuels and propellants for spacecraft. In-situ resource utilization (ISRU) is therefore considered vital to reduce the costs of a sustained presence on the Moon and to support the future human exploration of the solar system [2]. An important scientific goal for future robotic missions to the Moon is therefore the scouting of available water resources with payloads that are sensitive to signals of traces of water on the lunar surface.

The European Commission's LUVMI-X project (Lunar Volatiles Mobile Instrumentation - "Extended") is developing a mission scenario as well as payload and mobility breadboards for the detection of water and other volatiles in the lunar polar regions on a small, lightweight rover [3]. One of the instruments part of the LUVMI scenario is VOILA (Volatiles Identification by Laser Analysis), which uses LIBS (laser-induced break-down spectroscopy) to analyze the elemental composition of lunar surface materials. LIBS uses a pulsed laser to ablate material from an investigated target, which forms a bright plasma plume that can be analyzed spectroscopically to gain an emission spectrum of atomic and ionic emission lines of the elements in the target. The technique only requires optical access to its target and allows for quick measurements within a few seconds, making it well-suited for quick analyses of multiple targets in proximity of the rover [4]. LIBS was first used in space exploration by the US-French ChemCam instrument on NASA's Mars Science Laboratory mission with the CURIOSITY rover [5, 6], now followed by the improved SuperCam on NASA's Mars 2020 mission with the PERSEVERANCE rover [7]. Also China's Tianwen-1 Mars rover mission carries a LIBS [8], with landing planned for May 2021. 
The first LIBS instrument on the Moon was supposed to operate on board the Pragyan rover of India's Chandrayaan-2 mission [9], but the lander failed to achieve a soft landing in September 2019.

\section{INITIAL LAB EXPERIMENTS TO SUPPORT THE VOILA DESIGN}

Essential to the overall sizing of the VOILA instrument was a LIBS laboratory programme carried out at the DLR Institute of Optical Sensor Systems (DLR-OS) with the LIBS laboratory setups available at the institute. This campaign was critical because the VOILA LIBS will primarily be investigating soil targets rather than rocks that the LIBS instruments on Mars missions are optimized for, and because the near-vacuum environment on the lunar surface significantly affects the size and emissivity of the LIBS plasma plume.

The first LIBS setup that was used is capable of recording time-resolved high-resolution LIBS spectra in a broad wavelength range from $270 \mathrm{~nm}$ to $850 \mathrm{~nm}$, while the second LIBS setup is capable of measuring the time-resolved spatial distributions of selected plasma emissions, which is important to understand the spatial extent of the plasma in vacuum. Both use commercial Nd:YAG lasers firing into a vacuum chamber capable of reaching pressures lower than $10^{-4}$ mbar. The investigated samples were various lunar regolith simulants such as NU-LHT-2M, BP-1, JSC-1, Exolith LHS-1 and Exolith LMS-1. The high-resolution spectra of the first setup are recorded with an echelle spectrometer (LTB Aryelle Butterfly), while the spatially resolved spectra of the second setup are recorded with a monochromator (Andor Kymera 328i). In both cases an ICCD camera (Andor iStar) was used to achieve time resolution.
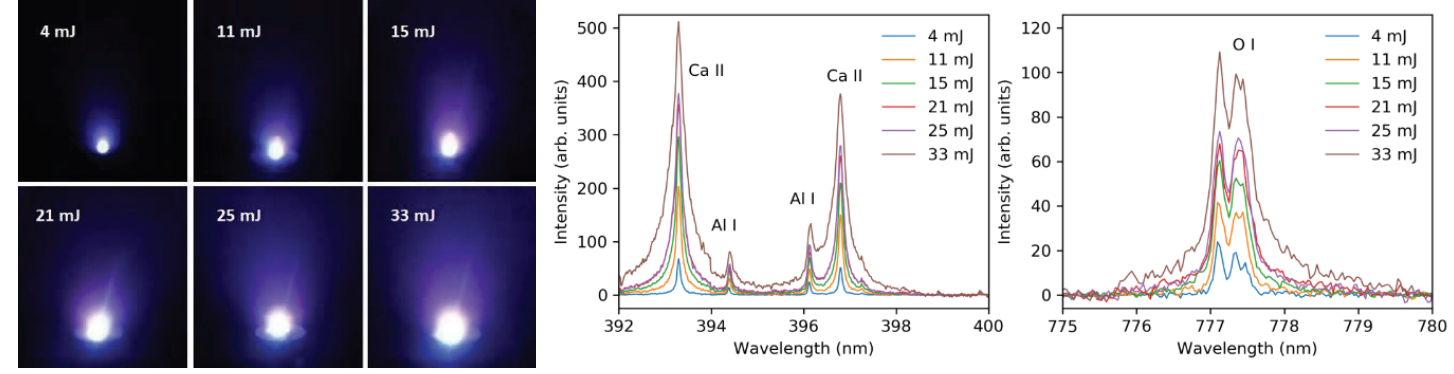

Figure 1, left: Laser-induced plasma on a BP-1 sample for different laser pulse energies at 10-4 mbar atmospheric pressure. Even at the lowest investigated pulse energies, the plasma is bright. Right: Plots showing spectral lines of Ca II, Al I, and O I in the recorded LIBS spectra. The line intensity and line broadening increase in accordance with the laser energy.

An important question to be answered by the preliminary lab programme was which pulse energy would be required to produce a suitably intense LIBS spectrum. Therefore, a study on the relation of the laser energy and the spectral intensity was conducted. Figure 1 shows images of the laser-produced plasma at different laser energies for a sample of BP-1 in lunar ambient conditions (left) as well as plots showing spectral lines of the corresponding LIBS spectra. While a strong increase of the line intensity can be observed for increasing laser energies, even the lowest investigated laser energy of $4 \mathrm{~mJ}$ produced a strong LIBS spectrum on the pressed pellets. For the highest laser energies, strong Stark broadening could be observed, which is unexpected at the low ambient pressure of $10^{-4}$ mbar that was used for these measurements. This indicates that even at low pressures, the laser-produced plasma features a high-density plasma center. The broadening at higher laser energies also limits the signal-to-noise ratio (SNR), so that the maximum SNR was already achieved at a laser pulse energy of $15 \mathrm{~mJ}$. 

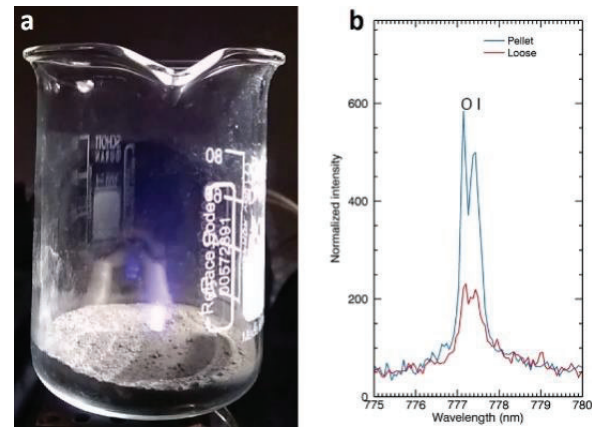

Figure 2: a) Image of the LIBS plasma of loose lunar regolith simulant grains (BP-1) at a pressure of 10-4 mbar. The laser energy was $15 \mathrm{~mJ} /$ pulse. b) Comparison of the intensity of the O I triplet at $777 \mathrm{~nm}$ for a pressed pellet of BP-1 and for loose BP-1 grains. The LIBS spectra were measured by averaging over 30 successive laser shots on the same spot on the sample.

In the next experiment, we compared the LIBS spectra that were measured for pressed pellets of lunar regolith simulants with those that were measured for loose grains. The pressed pellets are advantageous for laboratory studies due to their easier handling and better reproducibility of the measurement conditions, but loose grains are more similar to the regolith encountered on the lunar surface. Therefore, it is important to understand how the use of pressed pellets limits the validity of laboratory studies. Figure 2 shows an image of the laser-produced plasma in a Griffin beaker partially filled with loose BP-1 grains. The comparison of the LIBS spectra of these measurements to those of the pressed pellets indicates a significant decrease in intensity if the regolith is loose, which was also visually confirmed as the plasma was clearly less bright. However, for a laser energy of $15 \mathrm{~mJ} /$ pulse the LIBS spectrum was sufficient to detect most spectral lines of interest such as the O I triplet at $777 \mathrm{~nm}$. The SNR in the spectra can also be improved further by increasing the number of laser shots that are integrated to obtain a single spectrum.
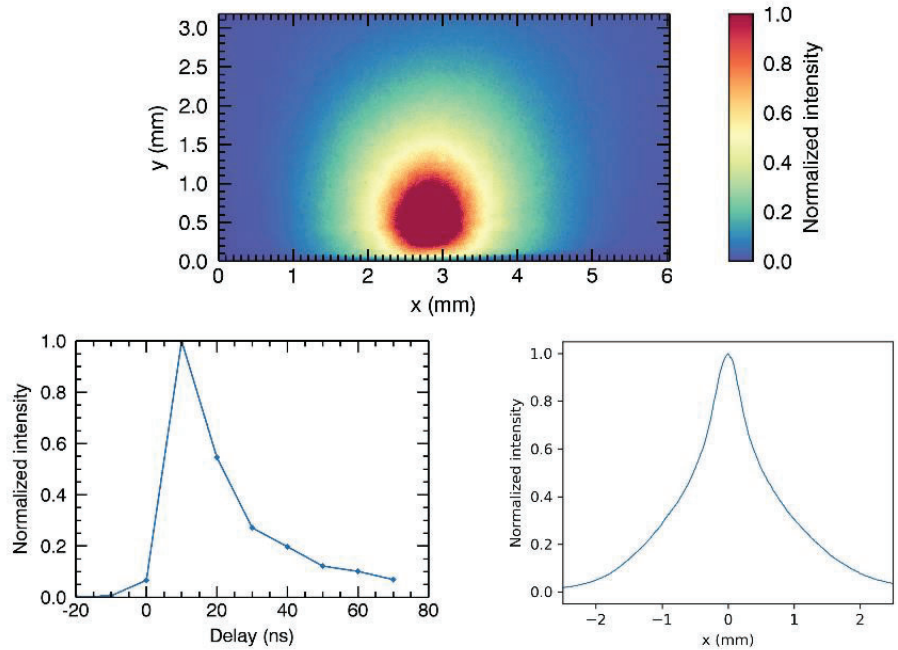

Figure 3, top: Spatial distribution of the time-integrated plasma emission of a NU-LHT-2M sample produced by a laser pulse of $15 \mathrm{~mJ}$ at $10^{-5}$ mbar atmospheric pressure. The intensity at the plasma center is saturated. Bottom left: Evolution of the spatially integrated plasma intensity over time. Bottom right: Intensity distribution along the horizontal axis. Most of the plasma emission is located in a

$2 \mathrm{~mm}$ region around the plasma center.

The spatial propagation of the LIBS plasma emissions in lunar ambient conditions is important because it can be used to optimize instrument parameters such as the field of view (FOV) and the design of the detection optics. Figure 3 shows the spatial distribution of the temporally and spectrally integrated plasma emissions for a NU-LHT-2M sample measured in low pressure of approximately $10^{-5} \mathrm{mbar}$ (top) as well as the temporal evolution of the plasma intensity (bottom left) and the intensity distribution along the horizontal axis (bottom right). It can be seen that, despite the low pressure and the lack of atmospheric confinement of the plasma plume, the emissive region of the plasma is still relatively large. Most of the 
emission is found in a region of $2 \mathrm{~mm}$ diameter around the plasma center, but the total emission extends out to a diameter of approximately $5 \mathrm{~mm}$. Considering that a large contribution to the central plasma intensity is the continuum emission that is not relevant for spectral analysis, the results indicate that a wide FOV of up to $5 \mathrm{~mm}$ may be beneficial to achieve optimum efficiency.
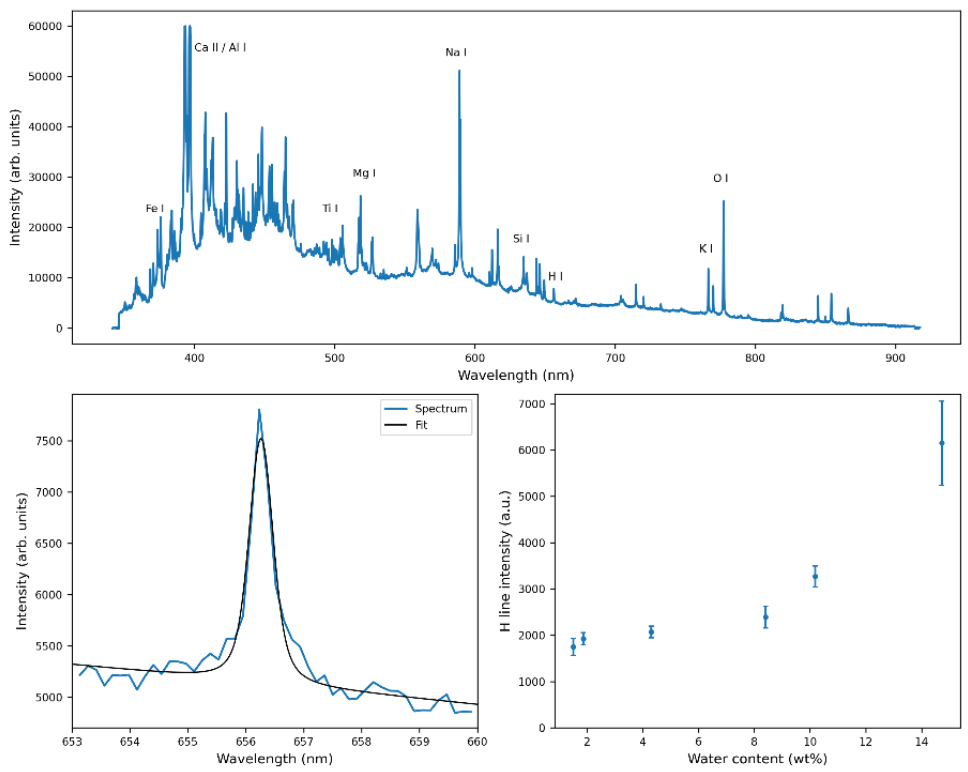

Figure 4, top: LIBS spectrum of a basalt/gypsum mixture with $6.3 \mathrm{wt} \%$ gypsum measured with a laser energy of $15 \mathrm{~mJ}$ and at a pressure of $10^{-4}$ mbar. Bottom left: Hydrogen peak in the same spectrum fitted with a Lorentzian line profile. Bottom right: $H$ I line intensity in dependence of $\mathrm{H}_{2} \mathrm{O}$ concentration in the samples from the basalt/gypsum test series. The offset of the line intensities at low $\mathrm{H}_{2} \mathrm{O}$ concentrations indicates a high presence of additional adsorbed water.

In addition to these general studies of the laser-induced plasma and its spectrum at near-vacuum pressures, the initial lab programme also included a preliminary calibration study to assess the feasibility of hydrogen detection and quantification and to estimate the limit of detection (LOD) for the hydrogen content. For this study, pressed pellets of finely-ground basalt mixed with gypsum $\left(\mathrm{CaSO}_{4} \cdot \mathrm{H}_{2} \mathrm{O}\right)$ were used, where the hydrated gypsum is supposed to simulate the presence of water ice. Six samples with gypsum concentrations between $6.3 \mathrm{wt} \%$ and $62 \mathrm{wt} \%$ were prepared.

While the experiment was originally performed with the high-resolution LIBS setup at DLR, it was subsequently repeated with an ad-hoc detection setup mounted to the same vacuum chamber. This ad-hoc setup more closely represented the VOILA design by using a compact Czerny-Turner spectrometer (Avantes AvaSpec-Mini) with a wavelength range from $340 \mathrm{~nm}$ to $900 \mathrm{~nm}$ at a spectral resolution of approximately $0.4 \mathrm{~nm}$. The plasma emission was collected with two lenses ( $f_{1}=200 \mathrm{~mm}$ and $f_{2}=60 \mathrm{~mm}$, respectively) and guided into a fiber that was connected to the spectrometer. Since the results using this setup are closer to the expected performance of the VOILA instrument, we will focus here on these results.

Figure 4 shows the LIBS spectrum that was obtained with this detection setup for the sample with the lowest gypsum concentration measured with a laser energy of $15 \mathrm{~mJ}$ in low ambient pressure of $10^{-4} \mathrm{mbar}$. Many spectral lines are covered by this wavelength range, including those of major rock-forming elements such as $\mathrm{Ca}, \mathrm{Mg}, \mathrm{Ti}, \mathrm{Si}, \mathrm{Fe}, \mathrm{Al}, \mathrm{K}$, and $\mathrm{O}$. The hydrogen line at $656.3 \mathrm{~nm}$ can also be observed and was fitted with a Lorentzian line profile (Figure 4 bottom left). From these fits, the intensity of the line was obtained and plotted in dependence of the water content in the sample based on the gypsum concentration (Figure 4 bottom right). It can be observed that the hydrogen line intensity increases for increasing water content, which supports the hypothesis that water quantification can be achieved with a hydrogen calibration curve. However, it can also be seen that no linear correlation is present and that there is an offset at low water concentrations, where the hydrogen line intensity is higher than it should be given the water supplied by the gypsum in the mixture. This indicates that adsorption of atmospheric water plays an important role and makes experimental studies with known hydrogen concentrations in the sample challenging. 
In summary, the initial test programme was able to provide important information for the design of the VOILA instrument. It also confirmed the robust detection of emissions of $\mathrm{H}$ and $\mathrm{O}$ from the samples for equivalent abundances of $\mathrm{H}_{2} \mathrm{O}$ upwards of $\sim 1.5 \mathrm{wt} \%$. Moreover, abundances of several major and minor rock forming elements contained in the target were also confidently detected. However, the results also confirmed that experimental studies on hydrogen quantification and detection limits are challenging due to the strong water adsorption that affects the actual hydrogen concentration in test samples. This will be an important issue that needs to be addressed in order to provide good data on the instrument capabilities at low water concentrations.

\section{INSTRUMENT OVERVIEW}

The sizing and design of the VOILA LIBS for future lunar polar missions is in part driven by its intended accommodation on a modest-sized rover, responding to the LUVMI scenario. Here, an optical front end consisting of the laser and an optical head (rigidly coupled to one another) is accommodated in the "raw" outside lunar environment of the carrier vehicle. Working range of the instrument is confined to $\sim 300 \ldots 500 \mathrm{~mm}$ range which results from the mounting position on the reference rover and the vehicle height. Laser energies and sensitivity of the receiving optics are balanced for this working distance, and the ambition is not to replicate a design like that of the ChamCam and SuperCam Mars LIBS instruments that are part of remote sensing suite of tools on 1,000 kg class Mars rovers to allow screening of the elemental composition of primarily rock outcrop from a stand-off distance of several meters. In the LUVMI scenario, available power levels, instrument aperture and mass budgets do not allow for working ranges this large.

In Figure 5 below, the instrument's block diagram is shown. The optical front end includes the LIBS laser and the optical head comprising laser beam shaping optics, a focusing mechanism to adjust to actual distance to the target, and the receiving optics. The whole front end assembly can be rotated along one axis in order to aim at different targets on the lunar surface in front of the LUVMI rover. Incoming light collected from the laser-induced plasma is coupled into an optical fiber that guides it into the spectrometer residing in the rover body. The spectrometer then analyzes the LIBS spectrum in the wavelength range from $350 \mathrm{~nm}$ to $790 \mathrm{~nm}$, which includes the H emission lines at $486.1 \mathrm{~nm}$ and $656.3 \mathrm{~nm}$ as well as the emission lines of major rock-forming elements such as $\mathrm{Ca}, \mathrm{Mg}, \mathrm{Si}, \mathrm{Fe}, \mathrm{Al}$, and O. Figure 6 shows a $\mathrm{CAD}$ model of the optical head of VOILA mounted at the front of the LUVMI-X rover's body.

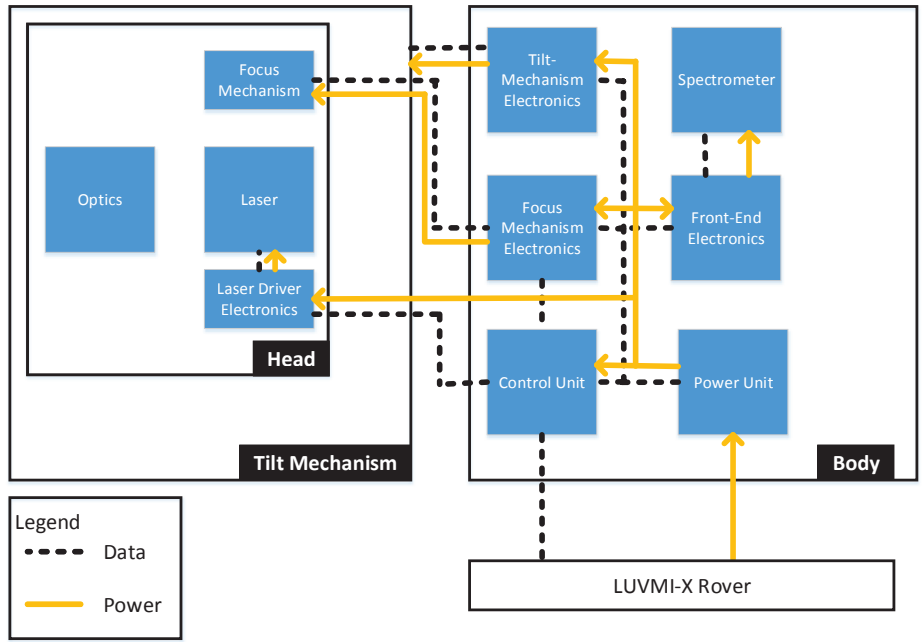

Figure 5: VOILA lunar LIBS block diagram 

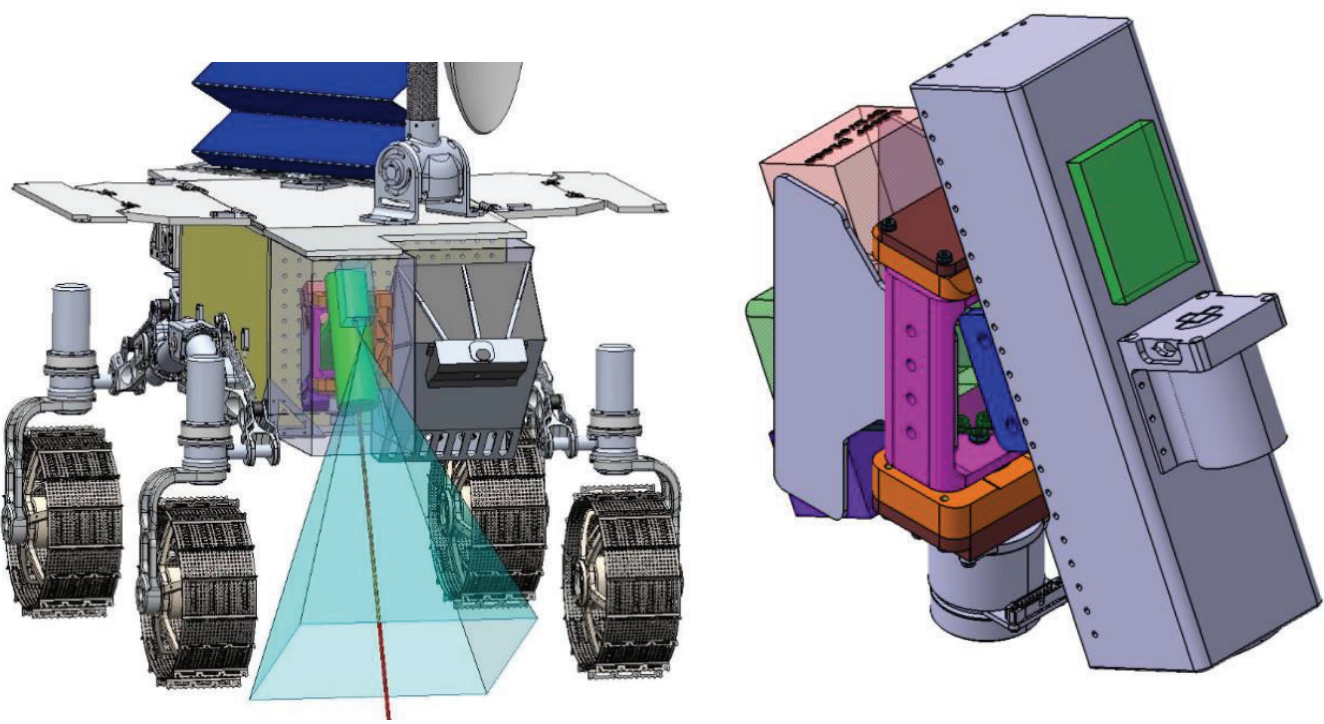

Figure 6: VOILA LIBS accommodated on LUVMI-X rover (left) and rover-external VOILA assembly (right) comprised of optical head with context imager (VOILA-Cam), tilt mechanism, laser, and laser driver electronics

The optical layout of the instrument is presented in Figure 7. The laser emits light at a wavelength of $1030 \mathrm{~nm}$ and its output is defined by a small beam expander with a magnification of approximately 2:1 resulting in a beam waist of $3 \mathrm{~mm}$ $\mathrm{x} 4.8 \mathrm{~mm}$ and a beam quality $\mathrm{M}^{2}$ better than 5.5 . The laser light reaches a dichroic mirror that reflects the laser light at $1030 \mathrm{~nm}$, but transmits light for lower wavelengths. The laser beam is further expanded by a 5:1 beam expander to a beam diameter of $15 \mathrm{~mm} \times 24 \mathrm{~mm}$ and focused onto regolith in a distance between $0.3 \mathrm{~m}$ and $0.5 \mathrm{~m}$. A focus mechanism allows to change the focal position within these extremes.

The laser spot diameter is less than $190 \mu \mathrm{m}$, which is sufficient for a laser with a power between $15 \mathrm{~mJ}$ and $25 \mathrm{~mJ}$ and a pulse duration of $8 \mathrm{~ns}$ to create a plasma bloom. The plasma is observed by the instrument. A circular area with a diameter larger than $5 \mathrm{~mm}$ of the plasma is focused onto an optical fibre bundle with a diameter of $300 \mu \mathrm{m}$. The collected light is transmitted through the fibre to the spectrometer in the body of the rover. For increased signal to noise ratio, the round fibre bundle is converted to a slit that the complete entrance slit with a size of $25 \mu \mathrm{m}$ is illuminated by the fibre. The spectrometer is of the type Crossed Czerny-Turner with a resolution better than $0.5 \mathrm{~nm}$ over a wavelength range of 350 $\mathrm{nm}$ to $780 \mathrm{~nm}$.

The centre fibre of the fibre bundle is separately mounted to a diode (LED or SLED) that emits light in the visible wavelength. The diode is switched on when the distance to the target shall be calculated. The diode transmits light through the glass fibre into the optical head that illuminates the target. The (blurry) spot is detected by the HazCams of the rover to calculate the distance between the VOILA instrument and the target. This value is then used by the focus mechanism to focus to the desired point. As the focus mechanism also focuses the diode light, a secondary evaluation for success in alignment may be done by the VOILACam, when imaging the target. The diode is located in the body of the rover to keep it in a less harsh thermal and radiation environment. 


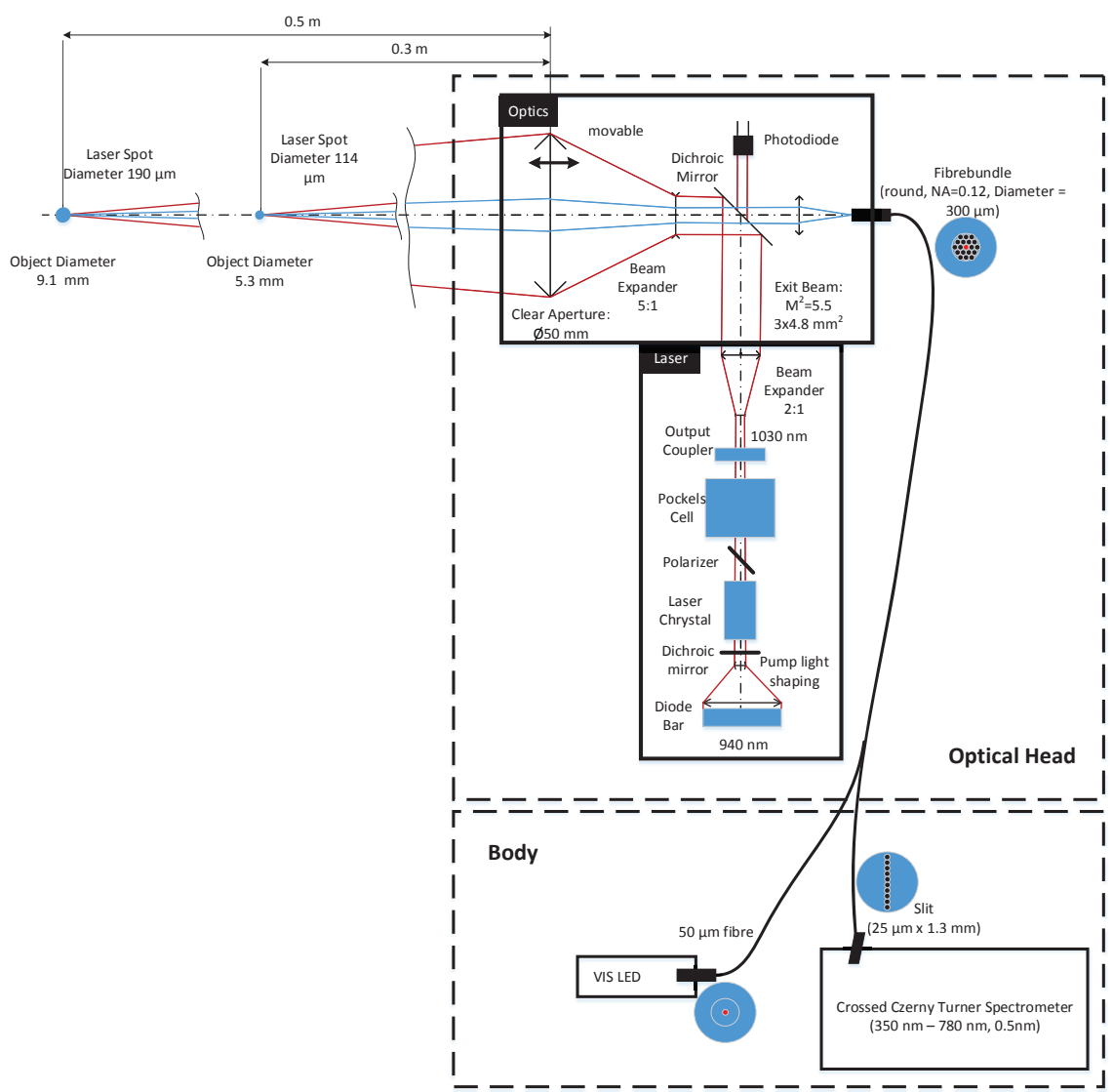

Figure 7: Optical architecture; the breakdown shows elements inside the rover body (spectrometer, detection, LED for target illumination as part of focusing concept)

\section{LASER DESIGN}

The laser system for the VOILA instrument is based on a diode-pumped actively Q-switched solid-state laser design. The general optical setup of the Q-switched laser system is shown in Figure 3.

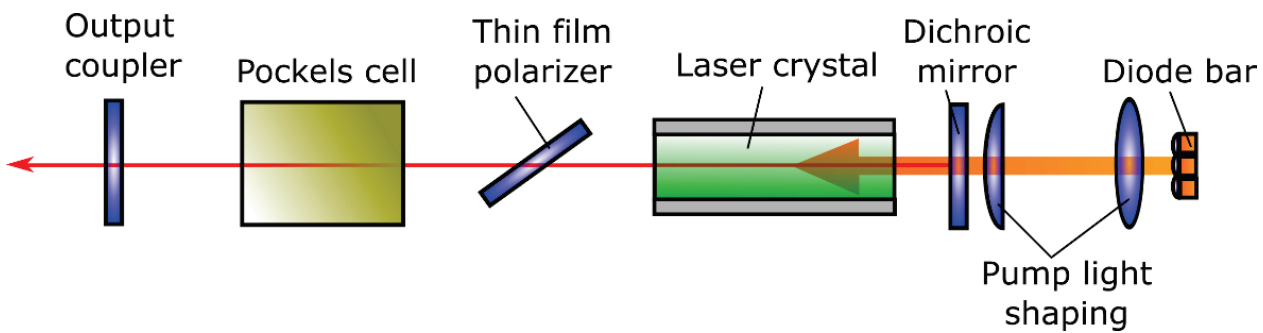

Figure 8: General optical setup of the actively Q-switched diode-pumped solid state laser.

The energy needed for the generation of the laser pulses within the laser resonator in the VOILA laser is provided by a laser diode bar (right side in Figure 8) operated in the quasi continuous wave mode (Q-CW). The light profile emitted from the laser diode bar is strongly elliptic. This profile is shaped by two cylindrical lenses and imaged into the laser crystal through a dichroic mirror, which is anti-reflection coated for the pump wavelength $(940 \mathrm{~nm})$ on the diode bar side and has 
a coating which is highly transmissive for the pump wavelength and highly reflecting for the laser wavelength (1030 $\mathrm{nm})$ on the laser crystal side.

As laser material, Yb:YAG was chosen. The long fluorescence lifetime of $\mathrm{Yb}$ :YAG of about 1 ms enables an efficient storage of the applied pump energy needed for the generation of the high-energy laser pulses. Furthermore, its three level scheme effectively inhibits parasitic lasing processes within the gain medium, which could otherwise occur due to the large accumulated gain.

For active Q-switching, a Barium borate $(\mathrm{Ba}(\mathrm{BO} 2) 2$ / $\mathrm{BBO})$ Pockels cell is chosen. The main reason for this choice is the relatively large optical damage threshold of $\mathrm{BBO}$, which is needed for this high energy laser system.

The design pulse repetition rate of the laser system is $10 \mathrm{~Hz}$. I.e., the temporal pulse spacing between two adjacent pulses will be $1 / 10 \mathrm{~Hz}=100 \mathrm{~ms}$. The design pulse duration of the laser system is $8 \mathrm{~ns}$ with a pulse energy of Ep $>15 \mathrm{~mJ}$.

Based on these design considerations, laboratory experiments were carried out successfully to deduce the best optical parameters (choice of lenses, resonator internal lengths, operating points etc.) for the realization of the needed optical output parameters. In a following design step, a laser breadboard system was designed and built to be used within the VOILA demonstration model, which will be described below.

\section{OPTICAL HEAD DESIGN}

The main sample type to be investigated on Moon is regolith. Preliminary tests in the laboratory have shown that LIBS will "drill" a small crater quite fast into the surface, which allows the plasma not to expand in all direction. This limits the visibility of the plasma by any other solid angles other than the LIBS excitation direction. Therefore a confocal system has been selected as optical design that uses a common front optics for the excitation path and the reception path.

The optical head has the functionality of expanding the laser and focusing it onto the sample for generation of the plasma and then collects the emitted light that is created by recombination of the ablated material. The receiving plasma light needs to be then separated from the excitation light through a dichroic beam splitter. Afterwards, the laser is focused into a fibre bundle for further transmission to the spectrograph. The optical head shall further focus the light from the laser head for working distances between $300 \mathrm{~mm}$ and $500 \mathrm{~mm}$. Furthermore, the laser pulse energy shall be monitored for housekeeping and error detection and shall provide a dot light source for focusing of the optical head.

The optics can be divided into the excitation and reception paths, consisting of:

- A beam expander optics: Expanding the laser beam and transmitting plasma light

- A dichroic beam splitter: In reflection for the laser beam and in transmission for the plasma light

- A front optics for focusing the laser beam and collecting the plasma light

- A fiber collimation optics: Coupling the collected plasma light from $350-790 \mathrm{~nm}$ to the fibre bundle

- A small fast focusing lens for the laser monitor diode behind the beam splitter, sampling the residual laser light transmitted through the dichroic ( $>$ OD3 reduction).

Further components of the LIBS optical head are:

- The mechanical structure of the optical head that is sealed against any Regolith dust, but allows for air exchange

- The laser head itself, that is hermetically sealed and air pressurized

- The pulsed pump driving electronics and high voltage electronics (Both need to be attached in vicinity to the laser head because of nanosecond timing)

- The focus mechanism

To fulfil the functionality of the optical head, the optical design consists of four elements. The collimation lens consists of two individual optical elements, similarly to the smaller elements of the beam expander (Beam expander 1 in Figure 9). The larger element of the beam expander (Beam Expander 2) that is also responsible for focusing the laser beam towards the target consists of three elements. Finally, the dichroic mirror between collimation lens and beam expander 1 shows the 
input coupling of the collimated laser beam. The raytrace in Fehler! Verweisquelle konnte nicht gefunden werden. 9 depicts the receiving path.

All optical elements have spherical surfaces and consist of Suprasil. This allows are fairly simple and cost effective manufacturing, while achieving reasonable performance over the large wavelength range of the receiving path from 350 $\mathrm{nm}$ to $790 \mathrm{~nm}$ as well as for the laser wavelength with $1030 \mathrm{~nm}$. To achieve the focusing of the receiving path and the excitation path, both small elements (collimation lens and beam expander lens 1) need to move to compensate the target distance. Thereby, the movement of both lenses is in the same direction and linked by a ratio of 1.2. The beam expander lens needs to travel by about $10 \mathrm{~mm}$, whereas the collimation lens moves in parallel about $12 \mathrm{~mm}$.

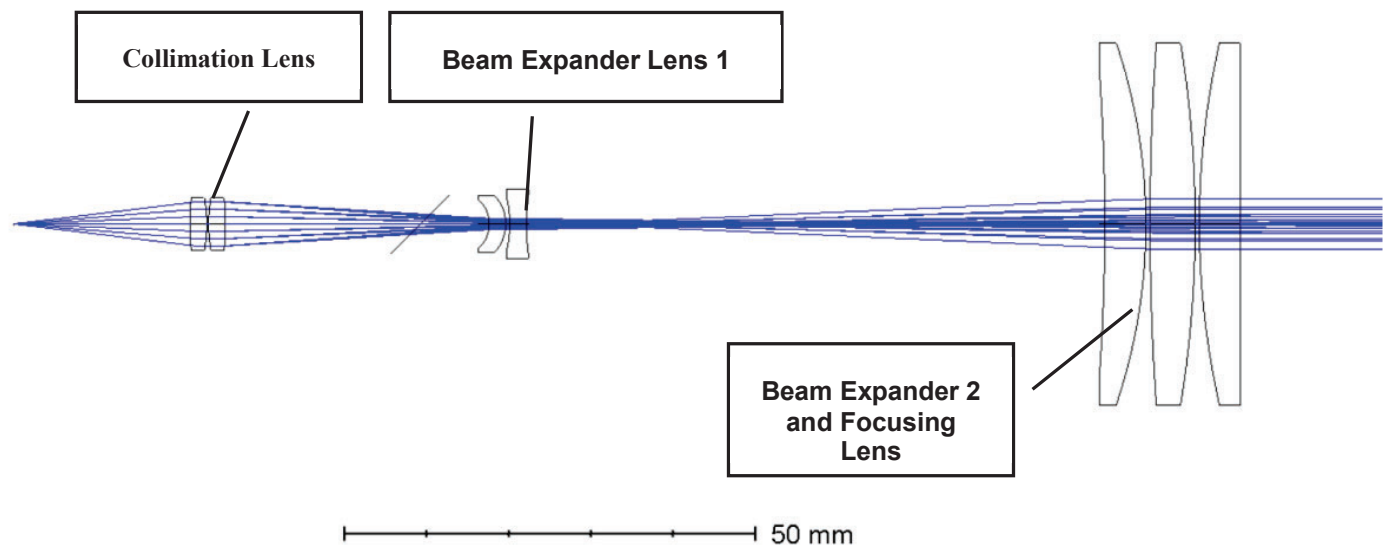

Figure 9: VOILA Optical Design

The optical head design and choice of materials is based upon a complete refractive design requiring that all lenses, windows and filters to be mounted adequately. Driving criteria are:

- the non-op (survival) temperature range together with the operational temperature

- $\quad$ possible thermal gradients inside the optical head

- the structural loads during start and landing loads

- $\quad$ radiation loads

To limit the thermal coefficient of expansion, thus linear and even local change of dimensions, and to be within the structural mass limits, a general TiGrade5 (Ti6Al4V) based design is preferred. The CTE of $\sim 8.6 \mathrm{ppm} / \mathrm{K}$ fits in average to many standard glasses. Using fused silica or other glasses/ crystals may require the use of local intermedia materials (Invar$1 \mathrm{ppm} / \mathrm{K}$, Kovar $-5 \mathrm{ppm} / \mathrm{K}$ ), pressure sealing, or even adequate kinematic mounts by introducing flexures. Latter ones shall be avoided as much a possible with respect to the limited available envelope. The temperature range is still with a certain limit which allows a hard mounted design. Additionally, fatigue or radiation damage issues are not expected due to the limited design lifetime of 14 days operational and 3 months of storage lifetime (in space).

A focus mechanism is required to adjust the laser's focus to the required distance and ensure a sufficiently high power density in the focus. The main challenge is to fit the focus mechanism in the limited available envelope and at the same time enable a large enough stroke and sufficient positioning accuracy. This can be achieved by various means. Often, the mechanism relies on compliant elements. This allows to achieve very precise positioning, usually at the cost of available stroke. Alternatively, a lead-screw based mechanism could be used which can achieve large strokes, but usually not quite the exceptional accuracy that is achievable with compliant mechanisms. Other possibilities include piezo actuators whose behavior is dominated by the control electronics, but generally achieve good accuracy at limited stroke. Additionally, a combination of the mentioned possibilities can be utilized at the cost of increased complexity.

For VOILA, movement of lens barrels is required, meaning that both sides of the optical elements need to remain "open", such that the optical path is not vignetted. Therefore, a main challenge is to arrange all elements within the limited and 
complex envelope. The optical system requires two lens doublets to be moved in the same direction, but not by the same amount. The ratio between the two always remains the same, such that both movements can be achieved with only one actuator driving the lens doublets via a different transmission. A simple system based on linear guides and lead screws seems preferable. A mechanism based on this operating principle is baseline for this application.

The presented focus mechanism relies on one stepper motor / gearbox combination to drive a segmented lead which features two sections with different lead. This allows to drive two nuts along the lead screw with different stroke/speed using only one actuator. The lead screw segments are connected at an interface that allows to rotate the segments with respect to each other. This is necessary to ensure the correct spacing of the two lens doublets. Via the lead screw and nut assembly, two carriages are moved along sliding rails. The lens doublets are supported on those carriages. The fiber end is mounted onto the fiber optics carriage that it remains in focus independently of the focus mechanism's position. End stop micro-switches provide a reference for step counting. A dedicated launch lock for the focus mechanism is not required.

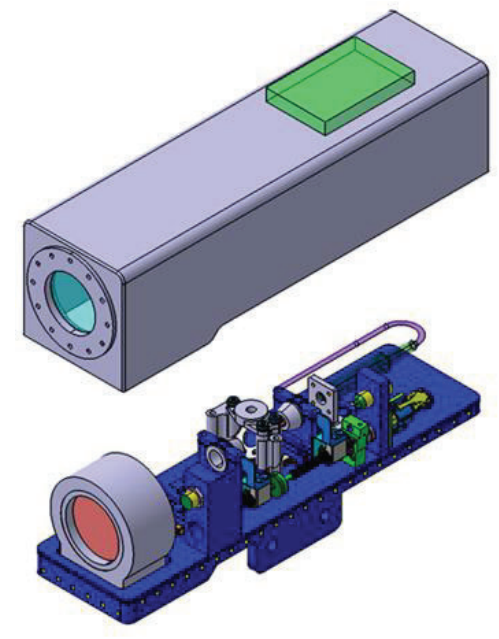

Figure 10: FM conceptual design of VOILA lunar LIBS optical head which combines laser beam shaping optics with plasma emission reception optics

\section{APPROACH TO SPECTROMETER AND DETECTION}

The spectrometer is designed to cover a wavelength range from $350 \mathrm{~nm}$ to $780 \mathrm{~nm}$ with a spectral resolution better than $0.5 \mathrm{~nm}$. Therefore, a grating with a grating groove density of about 600 lines $/ \mathrm{mm}$ is required. The UV-VIS spectrometer is based on the commercial QE-Pro or Maya spectrometer from Ocean Optics. The difference between the QE-Pro and the Maya spectrometers is that the QE-Pro is slightly longer to include the cooler of the detector. The optical bench is the same for both spectrometers. The spectrometer is a crossed-Czerny-Turner type spectrometer.

The main changes with respect to using a commercial system in a lunar environment regard the electronics and CCD. Two detector options are currently under investigation that differ in the amount of required adaption and thus in cost. The size of the optical bench allows to use either of them. The main reason to adopt the electronics and the CCD is to increase the radiation tolerance of the system. One important mitigation is to use multiple redundant detector pixel rows for the same spectral information. Thus, if a single pixel gets damaged and has a sharp increase in noise, it can be compensated for by other pixels that contain the same information.

One detector option is to use the detector S10420-1106 from Hamamatsu with 2048 x 64 pixels. Thus, in theory 64 redundant pixels can be used to detect the same spectral information. The detector is a standard detector for OceanOptics. The second option is to use the detector CCD42-10 from E2V with 2048 x 512 pixels. This detector has been used for ChemCAM and has thus space heritage. The decision on the final detector depends on the final mission parameters and a detailed radiation assessment. For a 600 lines $/ \mathrm{mm}$ grating, order sorting filters are not available as standard. It will be assessed whether it is necessary to implement an order sorting filter or whether the second order can be used to measure additional spectral lines. A custom order sorting filter will be developed for the flight model, if required. 
The spectrometer together with the instrument control electronics are located in the body of the rover to protect them from strong temperature variations and radiation damage. Figure 11 shows the preliminary design of the electronics unit and spectrometer box.

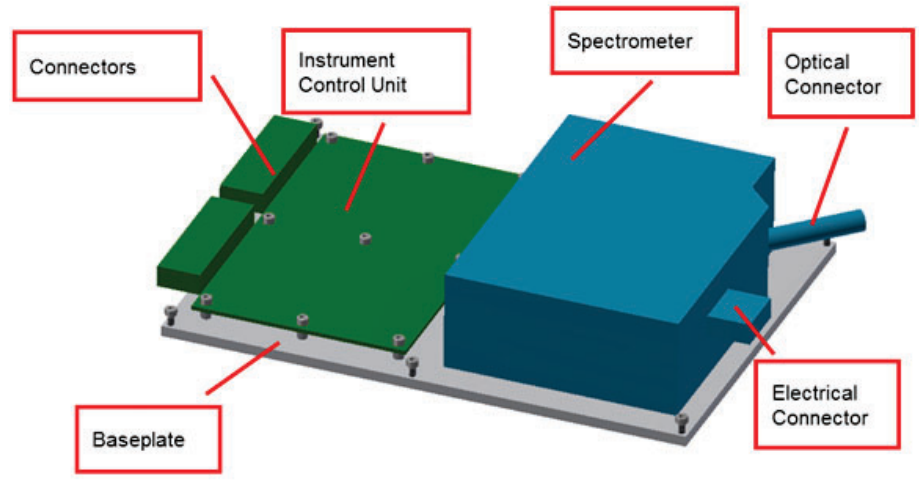

Figure 11: M arrangement of VOILA lunar LIBS instrument spectrometer and electronics unit (to reside in a single housing)

\section{THERMAL ASPECTS AND PRELIMINARY TECHNICAL BUDGETS}

A thermal assessment of the rover-external and rover-internal items of VOILA was performed through simplified thermal modeling, in order to specify expected parts temperature ranges and to determine required protective measures such as MLI and heating power to be provided by the rover for worst case situations.

Based on the selection of optical elements in the design, a first list a potentially temperature-critical components was derived and a literature research was conducted concerning available information with regards to space compatibility / qualification. This will lead to component-level low temperature exposure testing of selected, temperature-critical components.

Technical budgets and interfaces to the LUVMI rover have been defined for the VOILA FM concept in detail, capturing instrument mass, power requirements for various operating modes, estimated data volumes, thermal interfaces, and mounting requirements, among others.

The VOILA instrument is estimated with a mass of less than $6 \mathrm{~kg}$, including components inside and outside of the rover body. The power budget in Table 1 summarizes the required power for individual operational modes. The peak power of about $23 \mathrm{~W}$ is not reached in reality and is the theoretical value if all electrical components operate continuously and in parallel.

Table 1: Power budget for different modes

\begin{tabular}{|l|l|}
\hline Mode & Power [W] \\
\hline Peak Power & 22.9 \\
\hline Measurement Mode & 8.9 \\
\hline Idle Mode & 3.9 \\
\hline Survival Mode & 3.9 \\
\hline Recovery Mode & 3.9 \\
\hline
\end{tabular}




\section{LABORATOR DEMONSTRATION MODEL (DM)}

LUVMI-X being the framework project for the current development of the VOILA LIBS is a mission "architecture study" with concurrent development of breadboard versions on the scientific instruments the LUVMI rover would carry. For the LIBS, the LUVMI-X project scope includes a development model (DM) version which is suitable for laboratory conditions. For reasons of project effort, it was decided to create a DM whose components work at lab ambient conditions but with the LIBS laser firing through a window into a vacuum chamber hosting the respective sample (lunar regolith simulant). Likewise, also the plasma plume emission would be observed through a chamber window, but the receiving optics, spectrometer and detection again being located at lab ambient outside the vacuum chamber.

Regarding the breadboard model laser for the VOILA DM, the design was such, that reliable operation in the VOILA testbed at DLR-OS is possible. Figure 12 shows a CAD view of the laser design with the housing cover removed.

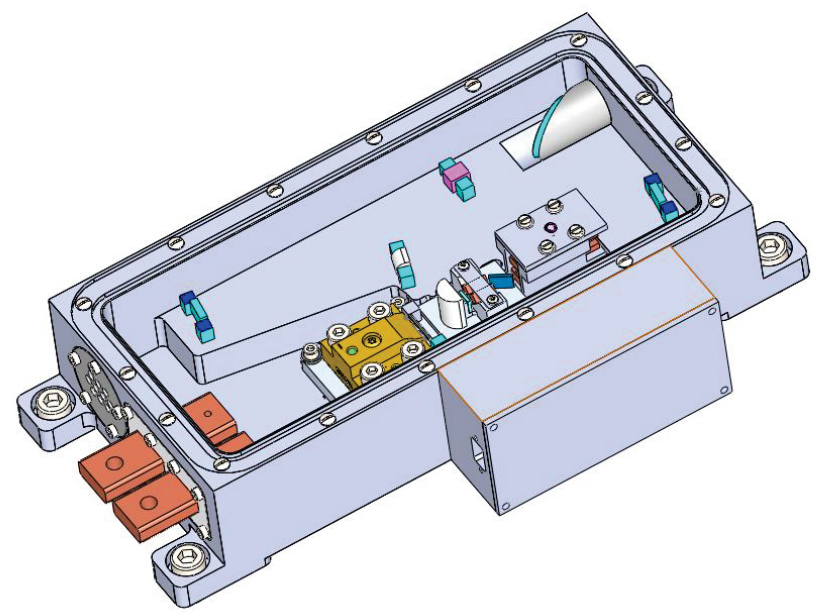

Figure 12: CAD view of the VOILA laser breadboard model.

The laser housing is dust-proof for protection of the delicate resonator optics from any dust particles for operation under normal laboratory conditions, but not hermetically sealed or pressure tight. Directly attached to the laser housing are the HV-electronics needed for Q-switched operation of the laser.

In Figure 13, the actual internal arrangement of the resonator optics and mechanics is shown with the resonator-internal laser beam path highlighted in green.

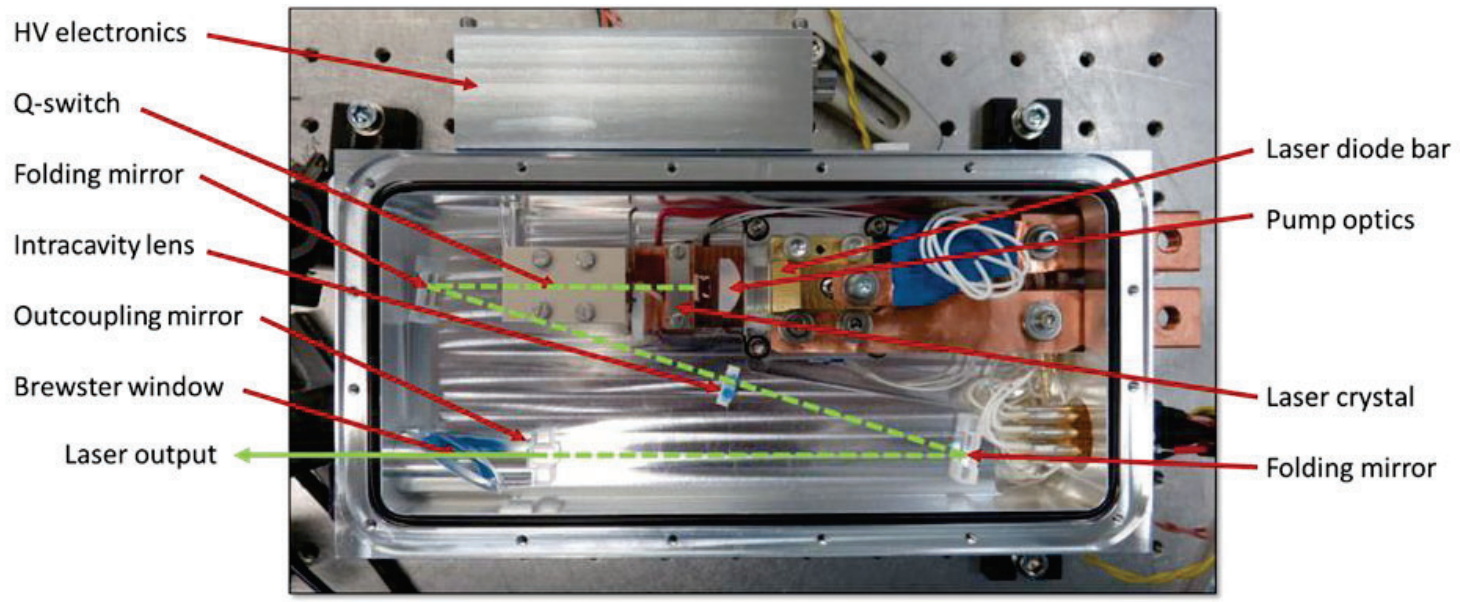

Figure 13: VOILA lunar LIBS breadboard laser source with housing cover removed. 
For easier DM integration, the laser was mounted on a height-adapting block to change the laser emission height to $100 \mathrm{~mm}$. This assembly was mounted with two temperature controllers on a common aluminum breadboard, see Figure 14. All other needed laser electronics were mounted into a 19"'-rack mounted box (not shown).
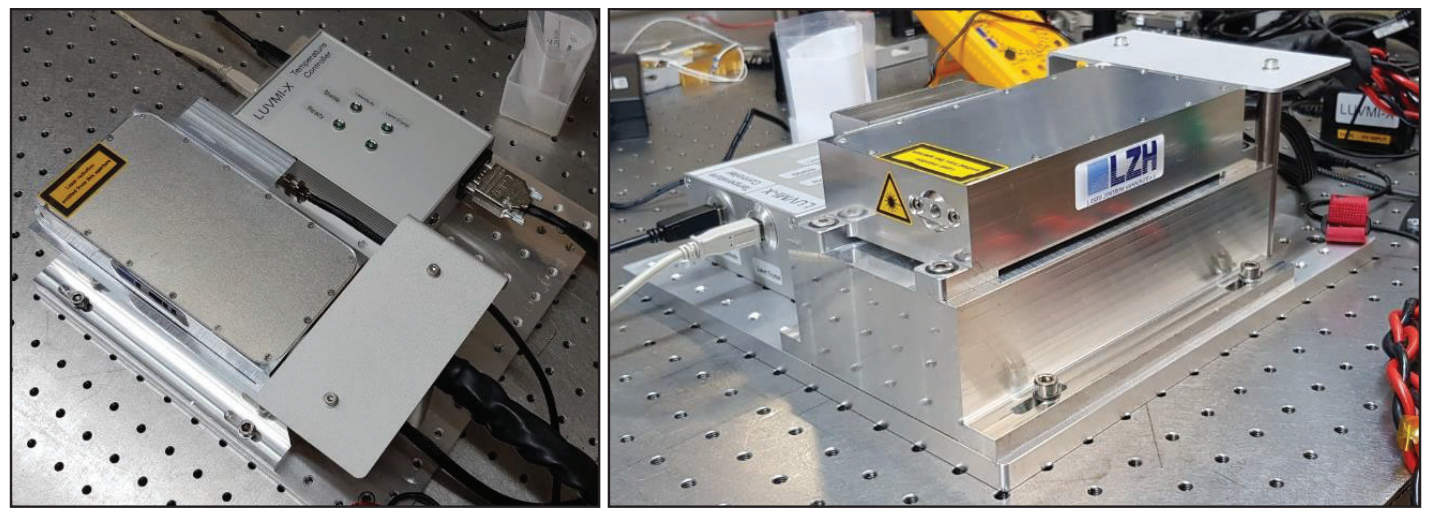

Figure 14: VOILA laser breadboard model ready for integration into the VOILA DM.

The laser system was thoroughly characterized and tested under normal laboratory conditions and it performed as designed. Pulses with a pulse length of $\sim 9 \mathrm{~ns}$ with a pulse energy $\mathrm{E}_{\mathrm{p}}>15 \mathrm{~mJ}$ were extracted at a repetition rate of $10 \mathrm{~Hz}$ and serve as the verification of the laser parameters for the LIBS process.

The breadboard laser system as it was mechanically realized is larger than the space allocated for the final flight model. However, a first conceptual mechanical design was realized demonstrating the fit of the laser concept into the allowed FM laser envelope. In addition, different options for future size reduction and increasing the ruggedness of the mechanical implementation are considered.

For the optical head DM, an optical design was developed which approximates the FM optical design but which can be built entirely from COTS elements. By changing the optical arrangement in selected aspects, and foregoing an active focusing for the DM fixed focus lab setup, it was possible to procure the DM optical head as an assembly of units from the same supplier, using standard laboratory mounting interfaces. 


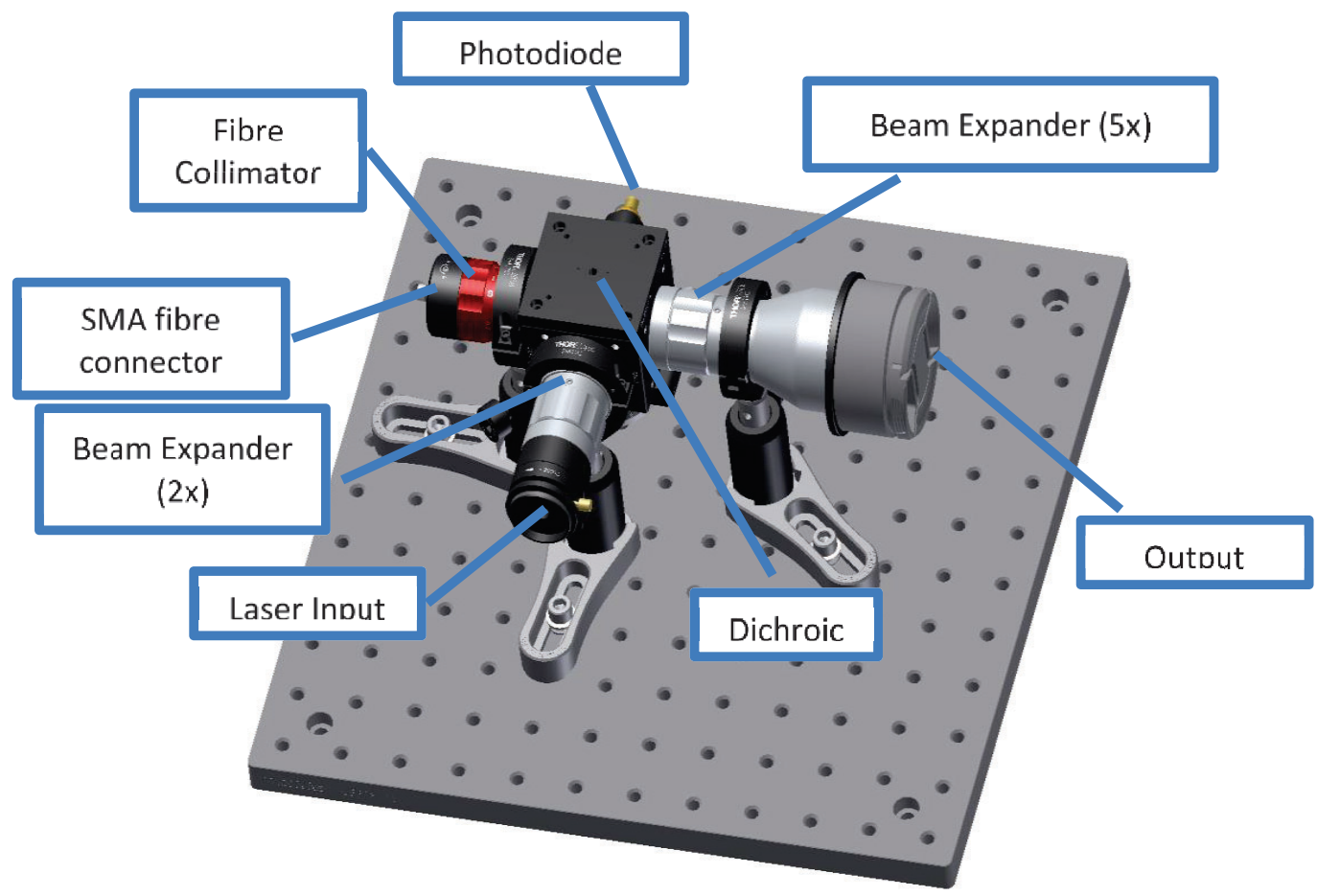

Figure 15: DM optical head

\section{EARLY LABORATORY RESULTS}

With the recently completed VOILA demonstration setup at DLR-OS (see Figure ), we were able to perform some early experiments to assess the performance of the system. All experiments were conducted in the VOILA vacuum chamber at pressures lower than $10^{-5} \mathrm{mbar}$, so that the measurement conditions are sufficiently close to those on the lunar surface. The breadboard VOILA instrument is mounted above the vacuum chamber with a fixed working distance of $400 \mathrm{~mm}$ and employs the optical head breadboard design developed by OHB and the laser developed by LZH. The laser operates at a wavelength of $1030 \mathrm{~nm}$ with a pulse energy of $17 \mathrm{~mJ}$ and a pulse duration of $7.8 \mathrm{~ns}$. The fiber-coupled spectrometer (Avantes AvaSpec-Mini) covers a wavelength range from $340 \mathrm{~nm}$ to $910 \mathrm{~nm}$ with a spectral resolution of about $0.4 \mathrm{~nm}$.
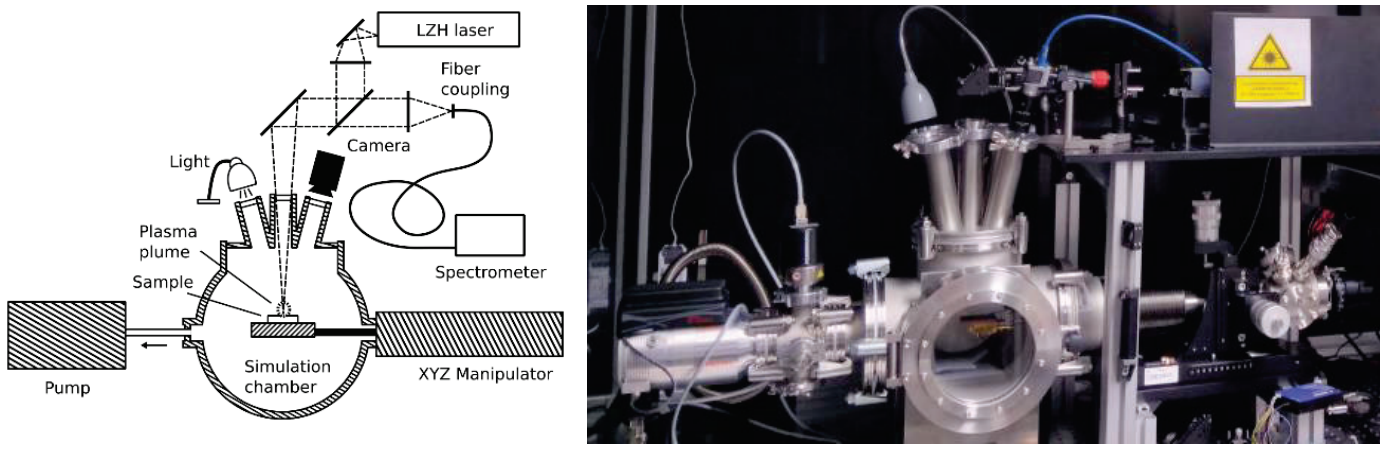

Figure 16, left: Schematic of the VOILA laboratory setup. Right: Image of the VOILA laboratory setup. 

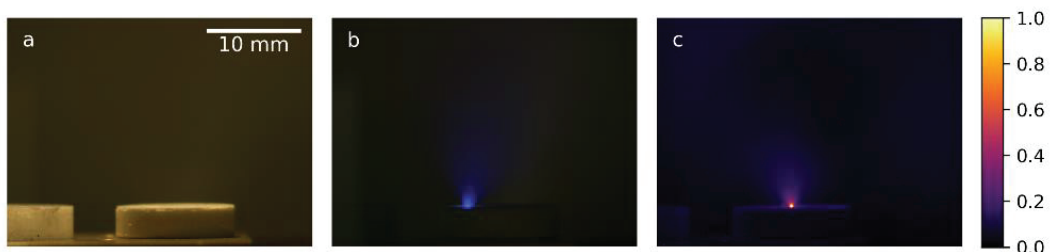

Figure 15: Gypsum sample on the sample stage inside the vacuum chamber before and during LIBS measurements. a) Image before LIBS measurement. b) Laser-induced plasma plume during LIBS measurement. c) False-color image of emission captured with a $656.3 \mathrm{~nm}$ bandpass filter.

Figure 15 shows a gypsum pellet (a) and images of the laser-induced plasma as a true-color image (b) and as a false-color image of the emission measured with a bandpass filter at $656.3 \mathrm{~nm}$ (c), which corresponds to the hydrogen emission line and to some continuum emission. The plasma has a bright spot at the ablation zone and lower-intensity emissions above the sample in a conical distribution with a diameter of several millimeters. Since the hydrogen emission is most relevant for the purposes of VOILA, this indicates that a large field of view is indeed preferable.

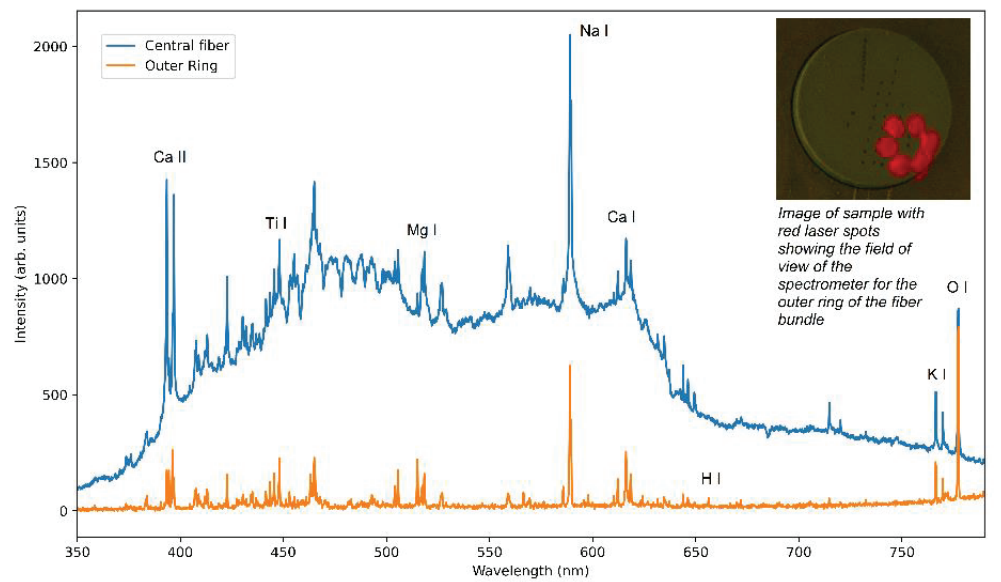

Figure 16: Comparison of LIBS spectra of the basalt/gypsum mixture with 6.3 wt\% gypsum measured with the central fiber and with the outer ring of a bifurcated fiber bundle. The spectrum recorded using the central fiber shows stronger continuum emission and the spectrum recorded with the outer ring shows the hydrogen line at $656 \mathrm{~nm}$, which cannot be observed in the other spectrum.

The LIBS spectra obtained with the VOILA instrument show the characteristic lines, but it has been observed that the continuum emission is higher than expected in the standard configuration where most of the collected plasma emission comes from the plasma center. Since the hydrogen emission has been shown to be widely distributed in the plasma, a study was done with different fibers coupling the emission into the spectrometer. A bifurcated fiber bundle with seven fibers in total was used where the fibers form a circle on the detection side, but where the central fiber is split off as a separate fiber on the other end. Figure 16 shows the comparison between the LIBS spectra that are obtained if the single central fiber is used for the light collection or if the outer ring consisting of six fibers is used. It can be observed that the continuum emission is much more intense in the case of the central fiber, whereas continuum emission is barely present if the outer ring is used. However, the hydrogen emission which could not be observed using the central fiber appeared in the spectrum of the outer ring. More studies are necessary to understand the underlying processes better, but the experiment indicates that occluding the plasma center could be beneficial for achieving a higher dynamic range. For now, the default configuration where the center of the plasma is not occluded will be used. 


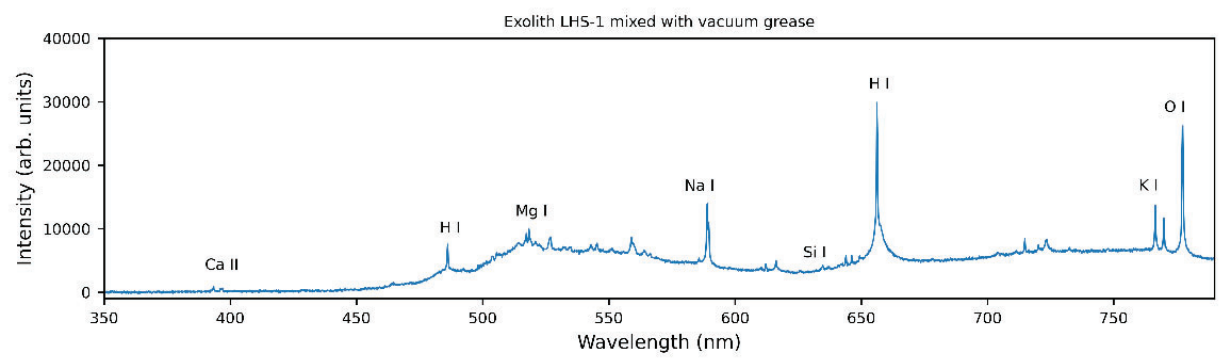

Figure 17: LIBS spectrum of lunar regolith simulant LHS-1 mixed with hydrogen-bearing vacuum grease. Both H I lines at $486 \mathrm{~nm}$ and at $656 \mathrm{~nm}$ can be observed clearly.

As a final experiment to investigate the capabilities of the completed VOILA setup, we measured the LIBS spectrum of a sample with a strong hydrogen signal. Since water quickly desorbs from the sample surface, we used vacuum grease that was mixed with the lunar regolith simulant Exolith LHS-1 to simulate a lunar target with a high water content. The LIBS spectrum of this sample can be seen in Figure 17. As expected, a very intense hydrogen line at $656 \mathrm{~nm}$ can be observed, but here the hydrogen line at $486 \mathrm{~nm}$ appears as well. Interestingly, the hydrogen line does not have the typical Lorentzian line profile, but seems to consist of a narrow peak and a broadened bottom. This could be caused by the superposition of a strong hydrogen emission from the dense early plasma, which will show significant Stark broadening, and the hydrogen emission from the late rarefied plasma where Stark broadening is negligible in comparison to the spectral resolution.

In summary, the results show that the VOILA instrument is capable of producing LIBS spectra that are suitable for the geochemical analysis of the lunar surface. There is still potential for further optimization, however, as LIBS in lunar ambient conditions provides special challenges that may be overcome by specifically designing the instrument to capture most of the available plasma emission while reducing the continuum emission that leads to a limitation of the dynamic range of the spectra. For future studies, this kind of optimization will be one important objective, with the other being the compilation of a VOILA spectra database of geologically relevant samples and of dedicated test series with different hydrogen concentrations.

\section{MISSION OPPORTUNITIES AND OUTLOOK}

The VOILA LIBS instrument being developed by us will be a versatile tool on future lunar landing missions. It is being developed specifically for vacuum environments and thus at the same time can become a candidate also for asteroid surface exploration missions. Currently, the baseline scenario is for VOILA to be accommodated on a lunar rover to support volatile scouting in the lunar regolith in the Moon's polar regions but the instrument can just as well be carried on static lander missions if the optical front head is mounted on a robotic arm for placement near the surface. Not only will VOILA be useful for volatile detection in the regolith but more generally for geochemistry as major rock forming elements are detected with good SNR.

Another relevant application is for the VOILA LIBS to be part of a lunar ISRU demonstration payload planned by the European Space Agency ESA: such payload would be a thermo-chemical processing facility to extract minerally-bound oxygen from the lunar regolith. As part of contextual measurements, elemental composition of the regolith is to be documented before the extraction process, and in this context we have infused the VOILA instrument into the current conceptual design of the ISRU demonstration payload.

The next step in our development logic is to develop an Engineering Model of the overall instrument.

*Lutz.Richter@ohb.de; phone+4981534002236; fax +4981534002940 


\section{REFERENCES}

[1] Li S. et al. (2018) PNAS, 36, 8907-8912.

[2] Anand M. et al. (2012) Planet. Space Sci., 74, 42-48.

[3] Gancet J. et al. (2019) ASTRA 2019.

[4] Knight A. K. et al. (2000) Appl. Spectrosc., 54, 331-340.

[5] Maurice S. et al. (2012) Space Sci. Rev., 170, 95-166.

[6] Wiens R. C. et al. (2012) Space Sci. Rev., 170, 167-227.

[7] Wiens R. C. et al. (2017) Spectroscopy, 32.

[8] Ren X. et al. (2018) EPSC 2018, Abstract EPSC2018-759.

[9] Laxmiprasad A. S. et al. (2013) Adv. Space Res., 52, 332-341. 